\title{
Personality Disorders in Childhood and Adolescence: Conceptual Challenges
}

\author{
Leslie C. Morey
}

Published online: 1 September 2010

(C) Springer Science+Business Media, LLC 2010

The papers by Salekin et al. (2010) and Tackett and Ostrov (2010) provide valuable data that illustrate many of the important determinations that shape the revision process of classification systems such as the American Psychiatric Association's Diagnostic and Statistical Manual. At the same time, the papers touch upon a number of important conceptual dilemmas that challenge the discipline. I offer the following comments to highlight areas that need greater exploration; I will note that it is certainly not a criticism of these articles that important questions in the field remain, at best, only partially answered.

\section{Behaviors vs. Traits as Defining Features of Problematic Personality}

The two articles each note the application and correlates of personality constructs (e.g. "relational aggression", "psychopathy") in children and adolescents that are presumably enduring features, or "traits", rather than referring to some specific behavior. Within the field of classification in mental health, there has been increasing interest in the utility of such personality traits as organizing and descriptive principles. As an example, Salekin et al. note that "The work that has been conducted on the psychopathy construct in youth has led the DSM-V workgroup to consider the possibility of incorporating psychopathy-like symptoms (callousness) as a potential specifier for conduct disorder (see Frick and Moffitt 2010)."

\section{C. Morey $(\bowtie)$}

Department of Psychology, Texas A\&M University,

MS 4235,

College Station, TX 77843-4235, USA

e-mail: 1morey@psych.tamu.edu
This trend represents a subtle but important shift in psychiatric nosology, one that invokes traits rather than behaviors in describing disorders. The earliest editions of the American Psychiatric Association's Diagnostic and Statistical Manual (DSM) provided definitions of disorders that were based upon rather vague symptom constellations. The DSM-III (American Psychiatric Association 1980) introduced a substantially different approach to representing all disorders, providing explicit diagnostic criteria in an effort to enhance the reliability of psychiatric diagnosis, which had appeared to be problematic in a number of studies. Because of the focus upon reliability, these diagnostic criteria often involved behavioral indicators, upon which agreement could be reached relatively easily. The DSM-III criteria for Antisocial Personality Disorder represent a clear example of this trend; diagnosing clinicians could potentially agree more readily on a criterion such as "three or more jobs in 5 years not accounted for by nature of job or economic or seasonal fluctuation" (American Psychiatric Association 1980, p. 320) than they could on a descriptor such as "individuals who are basically unsocialized", which was included in the DSM-II (American Psychological Association 1968, p. 43).

However, the following years witnessed somewhat of a pushback against these exclusively behavioral criteria, with the diagnosis of Antisocial Personality representing a prime example. Hare (e.g. 1985; Hare et al. 1991) in particular has been an advocate of including personality traits central to traditional conceptions of psychopathy. Hare et al. (1991) noted that the DSM-III/DSM-III-R behavioral criteria described a diagnostic category that was too heterogeneous, including most criminals and antisocial persons, but at the same time excluded those demonstrating the personality features of the psychopath but who had not exhibited the particular behaviors listed in the DSM. His review 
concluded that the "two factor" approach of his PCL-R measure - a structure that included both behavioral and trait elements of psychopathy - typically demonstrated superior predictive validity to the DSM-III definition (e.g., Hart et al. 1988; Harris et al. 1991).

By comparison to the DSM-III reliance upon explicit behavioral criteria, at least within the personality disorders, the pendulum has swung in the other direction in many ways. The DSM-5 Personality and Personality Disorders Work Group proposal describes problematic personality extensively in trait terms, rather than as constellations of problem behaviors. Such an approach would be in particularly marked contrast to traditional classification of childhood problems, which have focused almost exclusively on behavior constellations rather than on trait identifications. Thus, the DSM-5 proposal noted by Salekin et al. to introduce a partitioning of a behaviorally defined disorder (i.e., Conduct Disorder) based upon a trait attribution (i.e., Callousness) reflects a significant shift in thinking about the classification of these disorders.

The relative merits of behaviors vs. traits in characterizing individual differences has been hotly debated since the 1968 publication of Mischel's Personality and Assessment, and thus these issues have been covered with far greater consideration (c.f. Donnellan et al. 2009) than is possible here. It is important to recognize that behaviors and traits each have important roles to play in the classification of problems in living, although these roles can vary according to the nature and goals of the classification (e.g., Morey 1991a). For example, behavioral "symptoms" are typically the presenting complaint or concern for the person seeking mental health services, and as such provide appropriate targets for intervention. Furthermore, denoting such behaviors requires less inference, relative to traits, on the part of clinicians using the diagnostic system, which may lead to better interdiagnostician reliability. On the other hand, traits often provide superior long-term prediction of course and outcome (Morey et al. 2007). Also, trait concepts can allow clinicians to distinguish between individuals manifesting similar behaviors - such as aggressive behaviors - that appear to originate from different underlying sources - for example, instrumental patterns associated with callousness, as opposed to reactive patterns associated with poor affect modulation. While making such distinctions may require clinical inference, these patterns can have quite different outcomes (e.g., Pulkkinen 1996) and thus such distinctions are potentially quite useful in a classification of psychopathology.

Representing the construct of childhood psychopathy through a combination of the behavioral features of conduct disorder and the personality feature of callousness provides one example of a diagnostic "hybrid" that may allow these concepts to draw upon the useful information provided by both traits and behaviors. Doing so does pose some interesting conceptual challenges for the validation of these concepts. For example, during the development of the DSM-IV (Morey 1991a) I noted that the DSM was an interesting amalgam of hypothetical constructs and intervening variables, after MacCorquodale and Meehl (1948). For example, the classification itself provided a listing of hypothetical constructs (such as borderline personality, or schizophrenia) while the diagnostic criteria specified intervening variables thought to be useful markers of these constructs. The distinction in DSM-IV was fairly straightforward, even in the personality disorders section, in part because there was relatively little delineation of personality traits even though the personality disorders were putatively pathological manifestations of such traits. In actuality, the DSM-IV personality disorders more closely resembled a collection of constructs (such as Borderline personality) that were not really traits, to be identified through the use of behavioral indicators. In the DSM-5, it is likely that personality disorders will be delineated, at least in part, through the explicit use of traits - which themselves are hypothetical constructs, but which will in DSM-5 also serve as intervening variables for the definition of personality disorder constructs. Thus, there will be challenges posed for DSM-5 validation in considering the dual roles of traits as hypothetical constructs (e.g., do the measures of these traits have construct validity?) as well as in their role as intervening variables (e.g., does the specified combination of traits yield a valid representation of the overarching diagnostic construct?). Note that this is not to say that such a hybrid is problematic; for example, the PCL-R, in its two factor form, reflects a combination of behavioral and trait elements, and this combination has been used with good success as an indicator of the hypothetical construct of psychopathy. However, as noted by Skeem et al. (2010), when intervening variables are themselves hypothetical constructs, it is particularly important for the field to not confuse the indicators for the overarching construct itself.

\section{Levels of Analysis for Dimensional Models of Personality}

The investigations by both Salekin and by Tackett illustrate the applications of dimensional models of personality to represent concepts traditionally represented in the literature as categorical (e.g., psychopathy, early borderline personality). Thus, Salekin et al. describe measures of adolescent psychopathy in terms of domains (higher order factors) and facets (lower order factors) of the Five-Factor model, while Tackett and Ostrov represent relational aggression in a broader framework of internalizing and externalizing psychopathology - two "superfactors" that may character- 
ize common, underlying core psychopathological processes (Krueger 1999). This raises the issue of how best to represent these forms of problems in a dimensional system of psychopathology. In other words, how should the sometimes competing advantages of higher-order and lower-order features be balanced in a diagnostic system?

Many authors have invoked terms coined by biologist George Simpson (1945) who used "lumping" vs. "splitting" to characterize the conflict between parsimony and precision as strategic goals for taxonomy. Krueger (2002) suggests that "the broad-factor level of analysis and the facet level of analysis are complementary, rather than competing" and goes on to point out that "determining which level of analysis is appropriate for a particular situation is an empirical matter." However, as is typically the case for most empirical questions, the resulting answers may vary depending upon the conceptual framing of the question. For example, the very concept of "facets" as lower-order components in a hierarchical factorial model of personality is an interesting one. Conceptually, the notion of "factors" within the traditional exploratory factor analysis framework (the tradition from which most factorial models emerged) is that these factors identify communal variance among variables, a communality that would preclude interpretable "subfactors". Thus, finer gradations in such a model would presumably emerge as factors with lower eigenvalues, rather than as subcomponents of a factor with a large eigenvalue. For example, one of the first "hierarchical" factorial models was proposed by Eysenck and Eysenck (1963), who originally claimed that extraversion was composed of two positively correlated subfactors. However, in a subsequent refinement of this model, a third factor of psychoticism was identified that presumably eliminated the need to partition the extraversion factor. Similarly, in the development of the NEO-PI-R, Costa and McCrae (2008) noted the sometimes conflicting results provided by a goal of simple structure vs. a goal of facet elaboration of the five domains of the FFM.

It is intuitively appealing that lower order components of a dimensional model can lead to greater precision in specification of the relevant phenomena and superior measurement (Clark 2007; Samuel and Widiger 2008), and Salekin et al. echo this viewpoint by noting that "much more information can be gleaned from facet level analysis" (p. xx) in their paper. In fact, Block (1995) suggested that such advantages might render the higher-order dimensions unnecessary, noting that "If the global domains require articulation into more differentiating dimensions, it is because the summary labels and measures of the five broad, amalgamated factors are obscure, confounded, and perhaps even unacceptable in meaning." However, this is not necessarily the case. For example, Salekin et al. find that the facet level representation of the psychopathy measures differ in many ways across the two FFM instruments, while the representation at the domain levels are generally similar. While this could be a result of method variance tied to the specific instrument used, an alternative conclusion is that the identification of lower order facetsnot their measurement, but their structure-is simply less reliable than that of the higher order domains. There are a variety of findings in the literature to suggest such an interpretation. For example, with stepwise regression models such as those examined by Salekin et al., there is particular danger that overfitting may lead to the conclusion that models with many predictors will be superior to those with fewerand by definition there will be far more lower-order than higher-order dimensions. In fact, efforts to validate such models using cross-validation strategies (i.e., testing the generalization of fit models to other samples to examine shrinkage from overfitting) have found that lower-order facets provide little if any increment over predictions provided by higher order domains, a result found in both non-clinical (Grucza and Goldberg 2007) and clinical (Morey et al. 2007) data. Furthermore, although there is some consensus on the Five Factor Model as a replicable structure of higher order, there is not similar consensus on the structure of lower-order facets of personality (Block 1995).

It is worth noting that there are strategies to pursue greater precision and specification within factorially based dimensional models that do not involve facets of higher order factors. For example, as noted earlier, within the EFA framework it is always possible to extract and retain additional factors. The internalizing and externalizing "superfactors" noted by Tackett and Ostrov emerge with remarkable regularity as higher order components in psychopathology (e.g., Krueger and Markon 2006), dating back to Eysenck's (1947) initial efforts that extracted two orthogonal factors from psychiatric rating data - factors that he labels as neuroticism and extraversion, but which in their original definition converged with these two superfactors in many ways. Later, Eysenck sought to expand the reach of the model to address phenomena not well covered by these dimensions (e.g., psychosis) but did so through the extraction of a third factor, rather than through a partitioning of his first two factors.

A second strategy to enhance precision in the application of factorial models involves a focus upon configuration of higher order factors, rather than a partitioning into lower order factors. For example, although there are many studies examining how trait dimensions can be additively combined to represent mental health concepts, relatively few of these studies examine whether interactions between these dimensions play an important role. As one such example, the older concept of "primary psychopathy" (e.g., Lykken 1995) provides an example of the interactive influence of dimensional traits in understanding such concepts. The 
"primary psychopath" was one, in Eysenckian terms, who was unusually high in extraversion but unusually low is neuroticism. Thus, Salekin et al. note that Cleckley's classic description of psychopathy involved "having a complete absence of nervousness" (p. xx), but this characteristic in isolation would ordinarily be view as quite adaptive. From a trait interaction perspective, this feature only becomes salient in combination with the marked externalizing focus of extreme extroversion - a configuration that presumably gives rise to the problematic behaviors associated with psychopathy. However, to date there has been relatively little effort to examine the effects of constituent traits in interaction, as opposed to in linear combination (see Morey et al. 2000 for one exception). Even if only considering a high/medium/low characterization of the components of the Five-Factor Model, there would be $3^{5}$ or 243 interaction configurations between the component traits. Investigating the unique implications of these particular regions of the five-factor space, considering the configuration as more than just the "sum of the parts", is a "splitting" strategy that deserves more attention than it has received thus far.

\section{Information Sources in Assessment of Child and Adolescent Personality}

The two papers provide an interesting contrast in their measurement approach that nicely illustrate some of the complexities of diagnosis related to assessment methods. Participants in the Salekin et al. study, who were on average 15.2 years old, completed self-report measures of childhood psychopathy, interpersonal styles, and personality features. In contrast, the Tackett and Ostrov study, with participants having an average age of 9.9 years, had the majority of data provided by parents (both mother and father report), with one brief interview obtaining the report of the youths themselves. In general, this is characteristic of the literature; problems in childhood, having largely behaviorally based definitions, are typically assessed with behavior checklists completed by informants (e.g., parents, teachers, observers). On the other hand, problems in adulthood tend to be evaluating with method demonstrating greater reliance upon self-report, typically assessed either through questionnaire or through structured interview. In adolescence, there is presumably a transition between approaches, as both informant based (e.g., BASC, CBCL) and questionnaire-based (e.g., MMPI-A, PAI-A, PIY) methods are commonly used to assess problems in adolescence.

The potential impact of this transition is considerable, because both the literature review and the results provided by Tackett and Ostrov demonstrate that there is often considerable variability in constructs assessed in children, depending upon the source providing the data. For example, Tackett and Ostrov found that the correspondence between mother-report and child-report on the measure of relational aggression, while significant, was modest (roughly .30), underscoring the notion that using different sources of information can lead to appreciably different conclusions. If two methods indicate conflicting results, which should be trusted? In the Tackett and Ostrov study, the beta coefficients for parent reports were consistently significant predictors of measured internalizing and externalizing problems, while the child self-reports never emerged as a significant predictor. Although the correlated predictor variables could yield non-significant beta coefficients for the child self-report that could mask significant zero-order correlations, it is nonetheless the case that the child-self report was adding no unique prediction beyond that provided by parent reports. This could be interpreted to cast doubt on the validity of the child self-report, but this conclusion should be tempered by the shared method variance between parent report of aggression (relational and otherwise) and the CBCL measure of externalizing and internalizing, which is also based upon parent report. It is possible that, if the measures of externalizing and internalizing problems had been based upon child self-report, the pattern might well have been reversed.

This possibility is corroborated by the variety of significant correlations observed between youth psychopathy features and dimensions of personality in the study by Salekin et al., in which all measures involved youth selfreport. Thus, Tackett and Ostrov's results demonstrated that the nature and severity of perceived relational problems depend upon who is doing the perceiving; however, the question of which is most accurate will likely yield different answers, depending upon whose perception the validity criterion is based. This issue might be particularly salient for investigations like Salekin et al.'s, as some have targeted the construct of psychopathy as one for which selfreport is of dubious utility (Hare 1985); for example, if Cleckley's (1941) feature of "pathological lying" is indeed a central component of the construct, it is reasonable to be skeptical of the responses of such individuals concerning their honesty. However, this is not necessarily the case, as studies (e.g., Salekin et al. 1997; Buffington-Vollum et al. 2002; Walters and Duncan 2005) often find that antisocial features assessed by self-report measures such as the PAI (Morey 1991b) are as effective in predicting behavioral outcomes such as recidivism and violence as are observational or history based measures such as the PCL-R.

In assessment as in any research enterprise, it is best to have converging lines of evidence supporting the same conclusion. Ideally, ascertaining personality characteristics and problems would involve a triangulation process drawing information from self-report, observer report, informant report, as well as performance and laboratory 
based measures. Unfortunately, although converging assessment evidence provides for relatively straightforward interpretation, such convergence is not always obtained, as witnessed by Tackett and Ostrov's .30 correlation between mother-report and child-report of relational aggression. There is a critical need for further research on the manner in which data source moderates the validity of assessment. It has long been recognized within the child assessment literature that there tends to be greater convergence across information sources for externalizing problems than for internalizing problems (e.g., Edelbrock et al. 1986); although it is typically presumed that internalizing issues are best assessed through self-report, relatively little research has attempted to test this assumption. Along these lines, we conducted a recent comparative study of assessment modalities (Hopwood et al. 2008) using the prediction of longer-term outcome as a validity criterion that was assessed with both self- and interview data, and thus was methodologically balanced. We found that when self-report and interviewer-based assessment of borderline personality yielded conflicting information, self-report tended to perform better in assessing more experiential features that would not be directly accessible to an interviewer and would presumably require relatively greater clinical inference (e.g., identity disturbance, chronic emptiness). In contrast, interview judgments tended to perform better in the assessment of more observable or behavioral symptoms (e.g., self-harm, impulsivity). These results are consistent with the earlier results of investigators such as Edelbrock et al. (1986) in suggesting that, rather than concluding that one form of assessment will necessarily have superior validity to others, clinicians and researchers might give differential consideration to different assessment data sources depending on the nature of the characteristic being described.

\section{Developmental Continuity of Personality Problems}

Both of the papers identify the potential utility of considering traits as indicators of personality problems in childhood and adolescence-for Tackett and Ostrov, the potential role of relational aggression as a potential early indicator of borderline personality, for Salekin et al. the role of callousness and related features as early indicators of psychopathy. These reflect the increasing interest within the DSM-5 around the potential utility of trait determinations for distinguishing different forms of problems in childhood and adolescence. In earlier versions of the DSM, there was considerable ambivalence around the utility of personality disorder concepts prior to early adulthood. For example, the DSM-III listed "childhood equivalents" of several personality disorders that were to be used in individuals under the age of 18; only those disorders that had no such equivalent were to be used in children and adolescents, and then only in "unusual" circumstances. This implied discontinuity between children and adults was tempered somewhat in DSM-IV, where only Antisocial Personality was explicitly excluded from application to individuals under age 18 . However, DSM-IV still noted that the remaining disorders would typically not be recognizable until adolescence or early adulthood.

Despite this diagnostic ambivalence about the utility of personality concepts in children and adolescence, it is apparent that personality problems have a non-trivial genetic contribution (e.g., Livesley et al. 1998) and that personality and temperamental differences are evident in childhood and adolescence (Clark et al. 2000; Rothbart 2007). Thus, taking advantage of the potential utility of personality concepts for delineating problems of childhood and adolescence, either as diagnostic constructs or as important moderators of other diagnostic constructs, is an important line of investigation. There has been some hesitancy in doing so, as it is not necessarily the case that childhood problems seemingly related to personality variables such as shyness or aggressiveness will persist into adulthood (e.g., Parker and Asher 1987). However, it should also be noted that adult personality disorders will not necessarily persist, either (Grilo et al. 2004).

While there is likely to be significant trait continuity between childhood and adulthood, it is also clear that there are important developmental and maturational elements necessary to understand personality problems (Shiner 2009). As an example, the DSM-5 proposal for personality disorder identifies a core dimension of personality impairment common to all disorders - a dimension involving deficiencies in empathic capacity, failure to develop coherent and meaningful short-term and life goals; and inability to productively self-reflect (Bender et al., under review). While there are probably differences between children on such characteristics, there appear to be marked developmental differences between children and adults on these characteristics - using a common language example, an adult who is self-centered, non-reflective, and directionless might well be described by others as "childish". Thus, there is clearly more to be learned about these core manifestations of personality pathology and their developmental progressions. Each of the two papers makes a contribution to furthering our understanding of identity and interpersonal features in adolescents; as this understanding develops, so too will the utility of extending personality disorder concepts (as opposed to personality trait descriptors) downward into childhood and adolescence.

It would also be valuable to gain additional insight into the developmental progression of research findings indicating that, as noted earlier, different assessment methods that 
rely upon different information sources often show limited convergence. Typically, interpretation of observed discrepancies is made at the level of groups of individuals; for example, Cantwell et al. (1997) noted that a group of adolescents diagnosed via self-report will have higher prevalence of psychopathology than the same group of adolescents diagnosed using parent report methods. However, it would be interesting to investigate the implications of convergence or discordance on a particular individual with respect to long term outcome. In addition to reflecting problems with a particular assessment modality or incremental validity from differing modalities, discordant reports from parents, teachers, peers and the self might also be an indicator of a situational or contextually bound behavior. In other words, inconsistency across informants may reflect behaviors that are less a function of more stable personality process and more tied to a specific setting or relationship, and thus perhaps less likely to be manifest later in adulthood. Such a possibility was brought up by Bem and Allen (1974) in one of the earliest responses to Mischel's (1968) critique of trait psychology; Bem and Allen noted that results suggesting situational specificity of behavior were "predicated upon nomothetic rather than idiographic assumptions about the nature of individual differences" (1974, p. 508). In their view, a finding of situational inconsistency does not necessarily imply that individuals are inconsistent, it could also imply that particular participants do not agree with the researchers operationalization of the construct, or that the participants (including self and informant reporters) do not agree among themselves. Bem and Allen were thus one of the first to study Allport's (1937) "traitedness" concept, or the notion that certain traits are salient for some people, but not all. Thus, the traitedness approach treats consistency as a moderating rather than an outcome variable (e.g., Paunonen and Jackson 1985). Several operationalizations of traitedness have been studied, including interitem variability, scalability, construct similarity, and temporal response pattern stability (e.g., Biesanz et al. 1998). Extending this concept to include cross-method consistency of construct assessment, such as between self and other-report, would be an important direction for this approach.

\section{Summary}

The papers by Salekin et al. (2010) and Tackett and Ostrov (2010) touch upon a number of interesting issues related to assessment, diagnosis, and classification-issues that are quite topical given the development of the DSM-5. Unfortunately, these challenging issues will not be resolved prior to the publication of DSM-5, but articles such as these advance our understanding of these dilemmas and hold the promising of refining future classifications and their implementation.

\section{References}

Allport, G. W. (1937). Personality: An interpretation. New York: Henry Holt and Company.

American Psychiatric Association. (1968). Diagnostic and statistical manual of mental disorder (2nd ed.). Washington, DC: Author.

American Psychiatric Association. (1980). Diagnostic and statistical manual of mental disorders (3rd ed.). Washington, DC: APA.

Bem, D. J., \& Allen, A. (1974). On predicting some of the people some of the time: the search for cross-situational consistencies in behavior. Psychological Review, 81, 506-520.

Biesanz, J. C., West, S. G., \& Graziano, W. G. (1998). Moderators of selfother agreement: reconsidering temporal stability in personality. Journal of Personality and Social Psychology, 75, 467-477.

Block, J. (1995). A contrarian view of the five-factor approach to personality description. Psychological Bulletin, 117, 187-215.

Buffington-Vollum, J., Edens, J. F., Johnson, D. W., \& Johnson, J. K. (2002). Psychopathy as a predictor of institutional misbehavior among sex offenders: a prospective replication. Criminal Justice and Behavior, 29(5), 497-511.

Cantwell, D. P., Lewinsohn, R. M., Rohde, P., \& Seeley, J. R. (1997). Correspondence between adolescent report and parent report of psychiatric diagnostic data. Journal of the American Academy of Child and Adolescent Psychiatry, 36(5), 610-619.

Clark, L. A. (2007). Assessment and diagnosis of personality disorder: perennial issues and emerging conceptualization. Annual Review of Psychology, 58, 227-258.

Clark, L. A., Kochanska, G., \& Ready, R. (2000). Mothers' personality and its interaction with child temperament as predictors of parenting behavior. Journal of Personality and Social Psychology, 79, 274-285.

Cleckley, H. (1941). The mask of sanity. St. Louis: The C.V. Mosby Company.

Costa, P. T., Jr., \& McCrae, R. R. (2008). The Revised NEO Personality Inventory (NEO PI-R). In G. J. Boyle, G. Matthews, \& D. H. Saklofske (Eds.), SAGE handbook of personality theory and assessment. New York: Sage.

Donnellan, M. B., Lucas, R. E., \& Fleeson, W. (2009). Introduction to personality and assessment at age 40: reflections on the legacy of the person-situation debate and the future of person-situation integration. Journal of Research in Personality, 43, 117-119.

Edelbrock, C., Costello, A. J., Dulcan, M. K., Conover, N. C., \& Kala, R. (1986). Parent-child agreement on child psychiatric symptoms assessed via structured interview. Journal of Child Psychology and Psychiatry, 27, 181-190.

Eysenck, H. J. (1947). Dimensions of personality. London: Routledge \& Kegan Paul.

Eysenck, S. B. G., \& Eysenck, H. J. (1963). On the dual nature of extraversion. The British Journal of Social and Clinical Psychology, 2, 46-55.

Frick, P. J., \& Moffitt, T. E. (2010). A proposal to the DSM-V childhood disorders and the ADHD and disruptive behavior disorders work groups to include a specifier to the diagnosis of conduct disorder based on the presence of callous-unemotional traits. In American Psychiatric Association (Ed.), (pp. 1-36).

Grilo, C. M., Shea, M. T., Sanislow, C. A., Skodol, A. E., Gunderson, J. G., Stout, R. L., et al. (2004). Two-year stability and change in schizotypal, borderline, avoidant, and obsessive-compulsive personality disorders. Journal of Consulting and Clinical Psychology, 72, 767-775. 
Grucza, R. A., \& Goldberg, L. R. (2007). The comparative validity of 11 modern personality inventories: predictions of behavioral acts, informant reports, and clinical indicators. Journal of Personality Assessment, 89, 167-187.

Hare, R. D. (1985). Comparison of procedures for the assessment of psychopathy. Journal of Consulting and Clinical Psychology, 53, 7-16.

Hare, R. D., Hart, S. D., \& Harpur, T. J. (1991). Psychopathy and the DSM-IV criteria for antisocial personality disorder. Journal of Abnormal Psychology, 100, 391-398.

Harris, G. T., Rice, M. E., \& Cormier, C. (1991). Psychopathy and violent recidivism. Law and Human Behavior, 15, 625-637.

Hart, S. D., Kropp, P. R., \& Hare, R. D. (1988). Performance of male psychopaths following conditional release from prison. Journal of Consulting and Clinical Psychology, 56, 227-232.

Hopwood, C. J., Morey, L. C., Edelen, M. O., Shea, M. T., Grilo, C. M., Sanislow, C. A., et al. (2008). A comparison of interview and selfreport methods for the assessment of borderline personality disorder criteria. Psychological Assessment, 20(1), 81-85.

Krueger, R. F. (1999). The structure of common mental disorders. Archives of General Psychiatry, 56, 921-926.

Krueger, R. F. (2002). Psychometric perspectives on comorbidity. In J. E. Helzer \& J. J. Huziak (Eds.), Defining psychopathology in the 21st century: DSM-V and beyond. Washington, DC: American Psychiatric.

Krueger, R. F., \& Markon, K. E. (2006). Reinterpreting comorbidity: a model-based approach to understanding and classifying psychopathology. Annual Review of Clinical Psychology, 2, 111-133.

Livesley, W. J., Jang, K. L., \& Vernon, P. A. (1998). Phenotypic and genetic structure of traits delineating personality disorder. Archives of General Psychiatry, 55, 941-948.

Lykken, D. T. (1995). The antisocial personalities. Hillsdale: Erlbaum.

MacCorquodale, K., \& Meehl, P. E. (1948). On a distinction between hypothetical constructs and intervening variables. Psychological Review, 55, 95-107.

Mischel, W. (1968). Personality and assessment. New York: Wiley.

Morey, L. C. (1991a). The classification of mental disorder as a collection of hypothetical constructs. Journal of Abnormal Psychology, 100, 289-293.

Morey, L. C. (1991b). The personality assessment inventory professional manual. Odessa: Psychological Assessment Resources, Inc.

Morey, L. C., Gunderson, J. G., Quigley, B. A., \& Lyons, M. (2000). Dimensions and categories: the "big five" factors and the DSM personality disorders. Assessment, 7, 203-216.
Morey, L. C., Hopwood, C. J., Gunderson, J. G., Shea, M. T., Skodol, A. E., Grilo, C. M., et al. (2007). A comparison of personality disorder models. Psychological Medicine, 37, 983994.

Parker, J. G., \& Asher, S. R. (1987). Peer relations and later personal adjustment: are low-accepted children at risk? Psychological Bulletin, 102, 357-389.

Paunonen, S. V., \& Jackson, D. N. (1985). Idiographic measurement strategies for personality and prediction: some unredeemed promissory notes. Psychological Review, 92, 486-511.

Pulkkinen, L. (1996). Proactive and reactive aggression in early adolescence as precursors to anti- and prosocial behavior in young adults. Aggressive Behavior, 22(4), 241-257.

Rothbart, M. K. (2007). Temperament, development, and personality. Current Directions in Psychological Science, 16, 207-212.

Salekin, R. T., Rogers, R., \& Sewell, K. W. (1997). Construct validity of psychopathy in a female offender sample: a multitraitmultimethod evaluation. Journal of Abnormal Psychology, 106, $576-585$.

Salekin, R. T., Debus, S. A., \& Barker, E. D. (2010). Adolescent psychopathy and the five factor model: domain and facet analysis. Journal of Psychopathology and Behavioral Assessment. doi:10.1007/s10862-010-9192-7.

Samuel, D. B., \& Widiger, T. A. (2008). A meta-analytic review of the relationships between the five-factor model and DSM-IV-TR personality disorders: a facet level analysis. Clinical Psychology Review, 28, 1326-1342.

Shiner, R. L. (2009). The development of personality disorders: perspectives from normal personality development in childhood and adolescence. Development and Psychopathology, 21, $715-734$.

Simpson, G. G. (1945). The principles of classification and a classification of mammals. Bulletin of the American Museum of Natural History, 85, 1-350.

Skeem, J. L., \& Cooke, D. J. (2010). Is criminal behavior a central component of psychopathy? Conceptual directions for resolving the debate. Psychological Assessment, 22, 433-445.

Tackett, J. L., \& Ostrov, J. M. (2010). Measuring relational aggression in middle childhood in a multi-informant multi-method study. Journal of Psychopathology and Behavioral Assessment. doi:10.1007/s10862-010-9184-7.

Walters, G. D., \& Duncan, S. A. (2005). Use of the PCL-R and PAI to predict release outcome in inmates undergoing forensic evaluation. Journal of Forensic Psychiatry and Psychology, 16, 459-476. 